\title{
Intelligent tracking car design based on Fuzzy PID control YingChun Zhang ${ }^{1, \text { a }}$, Tao Hao ${ }^{1, b}$ and ZuoXun Wang ${ }^{1, c}$ \\ ${ }^{1}$ School of Electrical Engineering and Automation, Shandong Polytechnic University, Jinan, China \\ azyc_10@126.com, ${ }^{\mathrm{b}}$ haotao0803@163.com, ${ }^{\mathrm{c}}$ wangzuoxun@126.com
}

Keywords: PID control; STC89C52 microcontroller; sensor; DC motors

\begin{abstract}
A design method of intelligent car based on Fuzzy PID control algorithm was introduced here. By detecting various signals with sensors, both dc motors located on the left and right were controlled to achieve functions including track-finding automatically and overtake alternately. The proposed algorithm has advantages as a quick and accurate motor control, intelligent etc.
\end{abstract}

\section{Introduction}

This intelligent car was designed to attend the National Undergraduate Electronic Design Contest, and STC89C52 SCM was used as the total control chip, the signal collected by each sensor were sent to a stand-alone treatment (Fuzzy PID control, PWM calculation)[1]to control the L298 motor driver chip, and the two DC motors around the car can make the appropriate action, a single car track intelligently and two car overtake alternately. Materials of the intelligent car come from year's, with as, will be sent to so

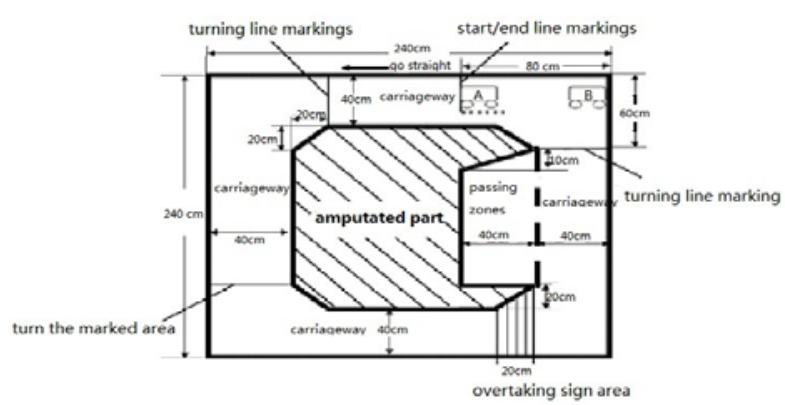

Fig.1 lane line

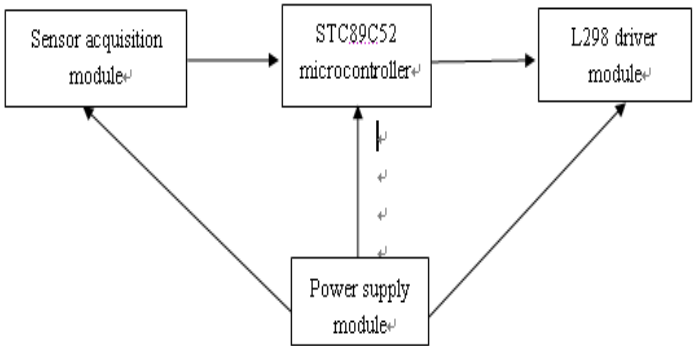

Fig.2 overall system block diagram

\section{System Design}

Microcontroller STC89C52 was adopted as the total control chip, and photodiode was used to collect the relevant signal as the input signal of the SCM. With the function of L298, the motor can do the appropriate action to realize track-finding and overtaking alternatively. The overall system was shown in Figure 2.

Sensor Acquisition Module

Photodiode was used as the sensor of the signal acquisition module[2], and the conduction of photodiode was converted to voltage signal change. Comparator LM393 was used to and compare photodiode signal acquisition and the result was sent to the microcontroller. In order to ensure the accuracy of the information collecting, three horizontal sensors on both sides of the car were installed to collect accurate information. Two rows of four vertical set of sensors were interposed in front of the bottom of the car to ensure the car turn accurately. Microcontroller which based on information collected performs the appropriate judgment to achieve the corresponding functions. Sensor acquisition module was shown in Figure 3. 


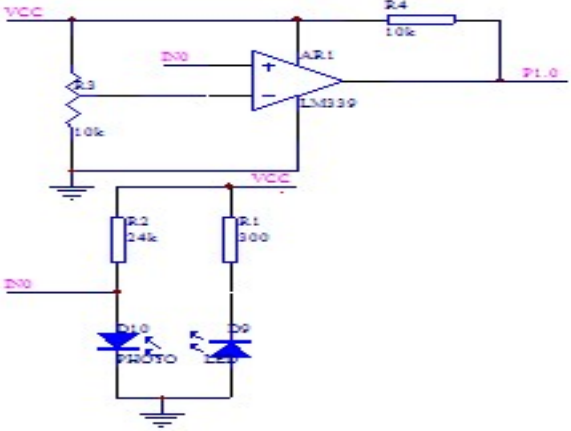

Fig.3 sensor acquisition module

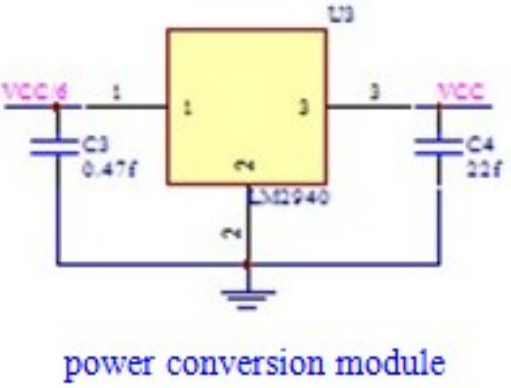

Fig.4 single-chip power supply module

Power Supply Module

Considered the supply of the motor speed and the controller, the power supply module was divided into two parts, the working voltage of the MCU module and the supply voltage of the motor drive modules L298. To ensure motor steady speed, the motor drive voltage was supplied by 6V battery power. To the single-chip, its requirement on supply voltage was $5 \mathrm{~V}$, here As the module taking into account, is: one is and LM2940 was used to converter 6V battery voltage to microcontroller for circuit simplicity. The voltage of Single-chip power supply module was shown in Figure 4.

Motor Drive Module and Single-chip control module

Thinking of the strong drive capability, simple circuit and the ability to drive two DC motors, L298 chip was used as the left and right DC motor driver chip of the car. Microcontroller calculated the appropriate PWM signal with Fuzzy PID theory to control and make the car straight-line driving, speed up, reduce speed, turn left, turn right and other functions. L298 driver circuit was shown in Figure 5.

Macro crystal STC89C52 was high-performance 8-bit controller with low power consumption, s, and it could meet the various requirements of the system. The control circuit was shown in Figure 6.

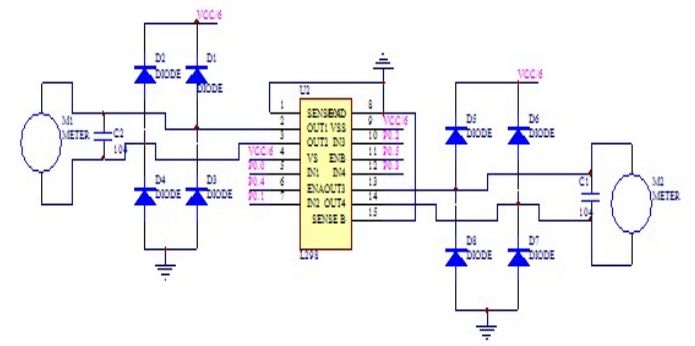

Fig.5 L298 driver circuit

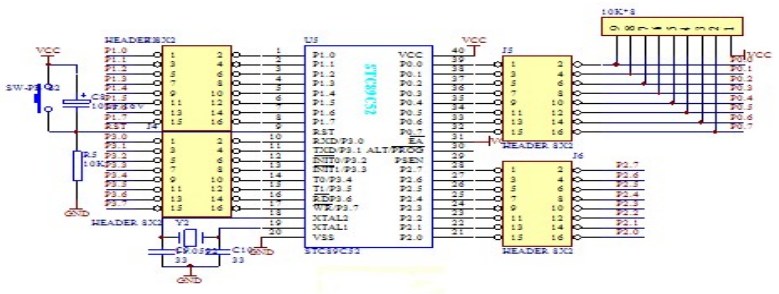

Fig.6 control circuit

\section{Software Implementation}

Program flow chart of single car track-finding was described as following. For a single car, the probes around both sides of the car to ensure the car to drive straightly, and it would be rectified to straight line with Fuzzy PID control when the car deviated the scheduled map-line. To achieve the fast and smooth adjustment of the car, Fuzzy PID parameters of the software can be adjusted conviently to guarantee the car drive along the predetermined straight line. By optimizing algorithm, the car can be accelerated when it goes straight and decelerate before it turning then accelerated when it came back to the straight line, which the time consumption on one-cycle can be minimum. Cycling program flow chart was in Figure 11. For two vehicles track-finding and overtaking alternatively, special turning mark was set in the passing line. The leading car would turn left and reduce the speed as soon as the special mark was detected until the mark was detected again and the car would recover its normal speed. For the lagging car, it would turn left and accelerate when special turning mark was realized until it overtook successfully and it would turn right and revolver its normal speed. When two vehicles were on straight line, the sending-receiving ultrasonic located in front of each car would determine which one was the leading and the lagging would overtake on the surpass lane. The program flow chart of two cars was shown in Figure 11. 
The key to decide the PID regulation performance was the selection of the parameter $\mathrm{Kp}, \mathrm{Ki}, \mathrm{Kd}$. Due to the impact on the system was interrelated and influenced each other, the choice of the traditional PID regulator relied on a lot of trial and experience. And when the object's state changed, the parameters of conventional PID regulator could not change, which caused the system unable to get satisfactory control. The PID regulator was a simulation of natural selection and evolution of the optimization algorithm, and it could change with the change of the object, so that the Fuzzy PID regulator parameters changed.

Fuzzy PID control at runtime based on the fuzzy relationship among the three parameters of PID controller with the deviation $\mathrm{E}$ and deviation change Ec detection of $\mathrm{E}$ and $\mathrm{C}$ (Cybernetic fields) by a predetermined relationship, the use of fuzzy inference methods, online change the PID the three parameters of the controller, the PID parameters could be self-tuning[3]. The control system block diagram was shown in Figure 7.

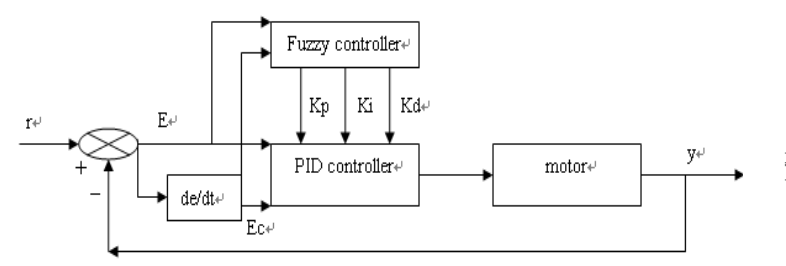

Fig.7 control algorithm system block diagram

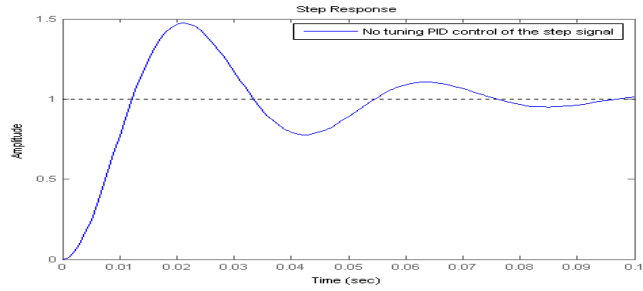

Fig.8 no tuning PID control of the step signal

Not a member of the fuzzy algorithm tuning PID parameter instability could be seen from Figure8. In order to prevent the control excessive, the square of the input was added to the objective function. The following formula was the parameter to select the optimal indicators.

$$
\mathrm{J}=\int_{0}^{\infty}\left(w_{1}|e(t)|+w_{2} u^{2}(t)\right) d t+w_{3} \cdot t_{u}
$$

In the formula $\mathrm{e}(\mathrm{t})$ was the systematic error, $\mathrm{u}(\mathrm{t})$ was the controller output, tu was the rise time, $w_{1}, w_{2}, w_{3}$ were the right value respectively [4,5]. In order to avoid overshoot, penalty function was adopted, that was, once the overshoot was found, the overshoot would be selected as the one of the best indicators, and the optimal index could be shown with formula (2).

$$
\text { If } \quad \mathrm{e}(\mathrm{t})<0 \quad \mathrm{~J}=\int_{0}^{\infty}\left(w_{1}|e(t)|+w_{2} u^{2}(t)+w_{4}|e(t)|\right) d t+w_{3} \cdot t_{u}
$$

In Figure 7, the parameters $\mathrm{Kp} \in(0,20), \mathrm{Ki}, \mathrm{Kd} \in(0,1)$, the optimized parameters $\mathrm{Kp}$ $=19.0832, \mathrm{Kd}=0.2434$, the performance index $\mathrm{J}=23.9936$. The tuning of Fuzzy PID control step response was shown below.
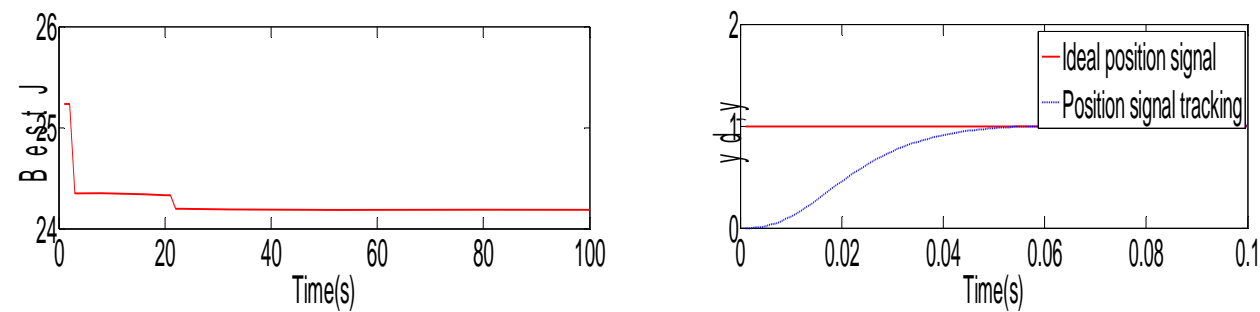

Fig.9 optimization of cost function value $\mathrm{J}$

Fig.10 step response of the turning PID

Using the new setting parameters to control motor movements in real time, precise control could be achieved. The car in the case of continuous conditions could keep moving to the specified location. 


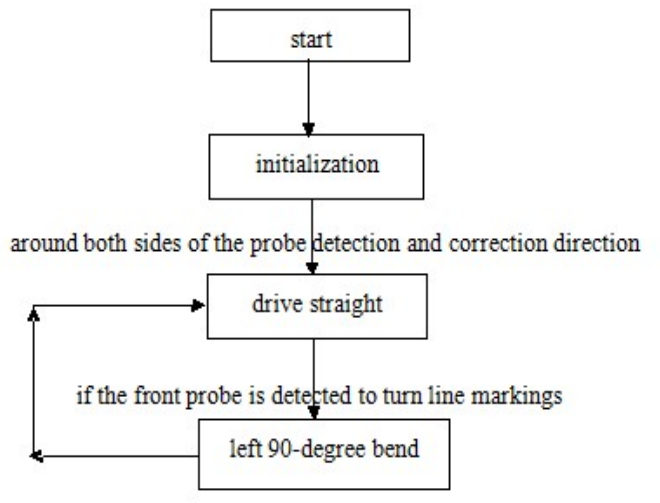

single car program flowchart

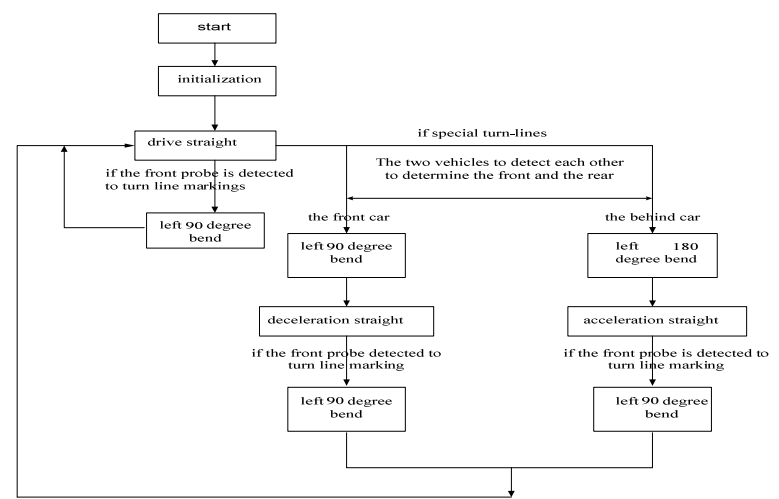

two-car program flowchart

Fig.11 Total Program flow chart

\section{Conclusion}

The circuit schematics of the intelligent car with track-finding and overtaking and software control of the corresponding algorithm were described here. The key to the hardware circuit was the accuracy of the information that the sensor collected, and the circuit needed to continue debugging. The sensor was installed in the proper height of the proper position in the actual operation so that the reaction would be accurately and sensitively. The software program design was the difficulty, a good program algorithm could ensure the car to achieve rapid, sensitive and accurate response. Here, PWM output was optimized with Fuzzy PID control theory, and it achieved the rapid control of the car movement with accurate response, thus vehicle track-finding, two vehicles track-finding and overtaking alternatively and other functions were achieved.

\section{References}

[1] Mei Chen, Yang Linlin, Li Xin, Xu Zhengrong DC speed control system Fuzzy / PID speed controller design. Electrical Drive Automation. (2008) .

[2] Long Zu Liang Ximing, Liu Can, Xu Yuebing Yang Lirong. Approximation accuracy of fuzzy controller based on the trapezoidal membership function, Hengyang Normal University.(2010).

[3] Kay realized. Wu Xun. Microcontroller C language program designed to self-study manual. Publishing House of Electronics Industry .(2008).

[4] Dong Chunxia first order inertia link fuzzy PID self-tuning control algorithm design. Higher vocational education (Tianjin Professional College).(2006 ).

[5] Jia Yi toward the vehicle active suspension fuzzy PID control simulation and analysis [J]. Tractors and farm vehicles.(2009). 